\title{
Comparison of Vital Capacity and Balance between Elderly Women and Young Women with Forward Head Postures According to the Use of Figure-8 brace
}

\author{
Eun-Kyung Kim \\ AMY Body-Mindfulness Research, Kimhae, Korea
}

\begin{abstract}
Purpose: To improve pulmonary function and decrease in balance ability with increasing forward head position and vertebral curvature, we applied Figure-8 brace to confirm the immediate effect on vital capacity and balance and to see if it is applicable.

Methods: A total of 34 elderly women aged 65 or older and young women in their 20s with FHP were screened to measure vital capacity, measuring the forced expiratory volume in 1 second (FEV1) and forced vital capacity (FVC), and measuring the foot pressure to see the change in balance. For statistical analysis, the difference between pre and post values was compared using pared t-test.

Results: As a result of vital capacity measurements, there was no significant difference between FEV1 and FVC for women over 65 years old ( $p>0.05)$. Young women in their 20s had no significant difference in FEV1 ( $p>0.05)$, and FVC had significant differences $(p<0.05)$. In measuring foot pressure to measure balance, both women aged 65 and above and young women in their 20s had a significant decrease in anterior foot pressure, and a significant increase in posterior foot pressure $(p<0.05)$.

Conclusion: The results of this study did not positively affect the vital capacity of elderly women with FHP. However, the significant increase in vital capacity of young women in their 20s suggests that contraction of the abdominal muscle is necessary during forced expiration. Therefore, it is believed that proper application and therapeutic interventions should be combined when applying Figure- 8 brace
\end{abstract}

Keywords: Pulmonary function, Forward head posture, Figure-8 brace

\section{Introduction}

Improper lifestyles and working conditions, and unstable postures cause compression and shear stress on the vertebrae. ${ }^{1}$ Also, involution of soft tissue and musculoskeletal system and trauma impairs effective regulation of spinal segments, and decreases the functional stability of spinal column. ${ }^{2}$ Sustained pressure in the anterior part of the vertebral leads to aggravation of vertebral kyphosis and constant overload in the neck tissue, resulting in a forward head posture (FHP). ${ }^{2-4}$ Such an postural dysfunctions increase with age. ${ }^{4,5}$ The thoracic curvature worsens with age and more in women than in men, especially.

FHP abnormally changes the anatomical structures of the cervical vertebra and thoracic vertebra according to the thoracic curvature, causing

Received Jul 11, 2019 Revised Aug 23,2019

Accepted Aug 29, 2019

Corresponding author Eun-Kyung Kim

E-mail kek74ing@naver.com changes in the thoracic cage and lung function, that resulted to chronic musculoskeletal system pain syndrome decreases the normal muscle breathing pattern and cause neck pain. ${ }^{4,7}$ Since the neck is the area that the respiratory assistant muscles refer to the function of the neck abnormalities affect the breathing function, ${ }^{7}$ a severe FHP causes shortening and weakening of the respiratory muscles, resulting in low respiratory circulation function and breathing capacity, it decreases forced expiratory volume in 1 second (FEV1), forced vital capacity (FVC).,8 To improve this, it is important to ease the increased vertical curvature to expand the thoracic cage and correct the breathing while increasing the mobility of the thoracic cage. ${ }^{4,7,8}$

The vertebral curvature caused by changes in the physiological ulterior motive causes morphology change in the spine trunk, and acts as a factor that increases the strain on the spine's multisegmental and trunk muscles

Copylight (C)2019 The Korean Society of Physical Therapy

This is an Open Access article distribute under the terms of the Creative Commons Attribution Non-commercial License (Http:// creativecommons.org/license/by-nc/4.o.) which permits unrestricted non-commercial use, distribution, and reproduction in any medium, provided the original work is properly cited. 
in a biomechanically standing position. ${ }^{2,4}$ Due to head and neck malalignment, increased abnormal curvature, lumbar lordosis and pelvis's anterior tilt, thereby changing the body's center of gravity position, thereby weakening the body's balance and ability to walk, thereby increasing the risk of fall. ${ }^{9,10}$

Therefore, therapeutic intervention is required to correct the FHP and vertebral curvature, maintain the correct posture and address muscle imbalance. Surgical treatment and conservation methods have been suggested as ways to improve excessive hyperkyphrosis. ${ }^{11}$ Surgical treatment is not recommended unless there is obstinate pain, severe disability, specific lung dysfunction, progressive neurological deficit. ${ }^{11}$ Instead, medical treatment, such as spinal orthoses, postural taping, intervention-based exercise therapy, to alleviate thoracic kyphosis is performed. ${ }^{1}$ Spine orthosis are effective in the conservative treatment and help with posture correction and reduce the sway of the body's center of mass to improve balance to prevent falls. ${ }^{1,10}$

Han et al. measured FVC and maximal volume ventilation (MVV) in young adults, and found that FHP was less comparable to FVC, FEV1, and Ratio of FVC to FEV1, MVV showed lower results than normal posture. ${ }^{12}$ Azadinia et al. improved posture stability and balance by wearing the posture-training support and spinomed orthosis which is thoracolumbaosacral orthosis ${ }^{13}$ Lee et al. For those with a round shoulder posture, measurement was made by wearing a Figure- 8 brace to correct the alignment of the scapula and improve the length of the pectoralis minor muscle to restore normal muscle activity around the scapula. ${ }^{14}$ This previous studies suggests that a may improve the FHP and the thoracic curvature.

Therefore, this study aims to improve the vertebral curvature by application of the Figure- 8 brace to young and elderly women with FHP and to investigate the immediate effect on the vital capacity and postural balance of each group.

\section{Materials and Methods}

\section{Subject's description}

The participants in this study sampled 34 women with forwardlooking head positions with a total of less than craniovertebral angle (CVA) $49^{\circ}$ in front of women aged 65 or older visiting S hospital located in Gwangju. The participants were divided into elderly women's groups and young women's groups, with 17 people each. All included patients understood the purpose of this study and provided written informed consent prior to their participation in the study in
Table 1. The characteristics of subjects

\begin{tabular}{lcc}
\hline Variable & Elderly group $(n=17)$ & 20's group $(n=17)$ \\
\hline Age (year) & $67.06 \pm 2.59$ & $20.82 \pm 1.70$ \\
height $(\mathrm{cm})$ & $155.8 \pm 3.95$ & $160.15 \pm 5.13$ \\
Weight $(\mathrm{kg})$ & $61.30 \pm .47$ & $53.74 \pm 8.67$ \\
CVA $\left(^{\circ}\right)$ & $43.03 \pm 2.11$ & $47.93 \pm 0.82$
\end{tabular}

Values are presented as mean \pm standard deviation, CVA: craniovertebral angle.

accordance with the ethical standards of the Declaration of Helsinki. Exclusion criteria of study subjects are as follows: 1) people with musculoskeletal disorders in the last 6 months, 2) severe spinal instability or osteoporosis, 3) cardiovascular or peripheral vascular disease, 4) history of pulmonary disease, 5) past operation history with leg, ankle and feet and problem of postural balance. Before participating in the experiment, all participants were fully informed about the experiment, understood its purpose, and voluntarily agreed to participate before the experiment. The general characteristics of the subjects are as follows (Table 1).

\section{Research procedure}

To screen the participants, all patients were instructed to sit comfortably in a chair and to watch a point in front of the patients to maintain a natural head posture (NHP) and to prevent postural change by visual activity. To measure CVA, 7th cervical vertebra and tragus were identified by manual palpitation, and sitting posture was taken using a camera at a distance of $2 \mathrm{~m}$ from the participant. The CVA was defined by the angle that consists of a horizontal line at $90^{\circ}$ to the vertical line passing through the 7 th cervical vertebra and an oblique line passing through 7 th cervical vertebra and tragus, using NIH Image J 1.32 for Windows (National Institutes of Health, USA) image processing program. CVA is a typical indicator of FHP, and the smaller degree of CVA, the greater the lower cervical vertebra flexion and the increase in FHP. ${ }^{15,16}$ CVA measurements were taken three times and the average value was adopted as the measured value.

In order to correct the vertebral curvature of selected FHP participants, Figure- 8 brace (Refarm, Korea) were applied across the bilateral axilla, coracoid process, and center of thoracic cage for each participant. To correct the vertebral curvature and position of scapula, the brace was applied to each participant using a pull-adjustable strap, which can be adjusted from 28 to 48 inches, to fit the torso width and apply sufficient tension. ${ }^{17}$ Detailed explanations were provided to familiarize with the experimental 
procedures and experiments before the measurement, and the measurement order of each participant was randomized. Before the measurement, accommodation procedure for Figure- 8 brace was performed at $10 \mathrm{~min}$ for each participant. The results of each measurement were measured three times and calculated as the average value.

\section{Measuring tool}

\section{1) Pulmonary function test}

FEV1 and FVC were measured using a spirometry (ML3500 MK6, $\mathrm{UK})$ to measure changes in vital capacity before and after wearing an Figure- 8 brace. The vital capacity test method was implemented in accordance with the manual of the American Association of Thoracic Society in the following manner. In order to prevent air from leaking during the measurement in the sitting position, the subjects were measured by blocking the nose with one hand, putting the disposable mouthpiece into the oral cavity for about $2 \mathrm{~cm}$, and performing three normal breathing exercises, requiring the maximum inspiration to match the examiner's verbal signals and descriptions, and continuously expiration for about 6 seconds until air is exhausted. FEV1 and FVC was measured during the maximum exhalation of participants and the fastest and strongest exhalation for a long time after maximum inhalation of participants, respectively. ${ }^{18}$ Measurements were performed three times before and after wear Figure- 8 brace, and each measurement was required to take a five-minute break. The average value of the resulting value was used as data.

\section{2) Balance measurement}

Foot plantar pressure measurement system (FDM-S, Zebris Medical GrmbH, Germany) were used to measure the balance of the subjects. This measurement tool is a device that has a pressure detector of 2,560 built into the force plate 64 balance of the subjects. This measuremenet tool is a devWinFM software. The method of measurement was to take off the shoes and socks, put both feet on the force plate, and watch the marking point located $3 \mathrm{~m}$ ahead with the arms laid down comfortably beside the trunk, and measure the plantar pressure. Measurement frequency setting was $50 \mathrm{~Hz}$ and measurement time was 10 seconds, measuring the ratio of the foot pressure distribution at the anterior and posterior of the plantar. ${ }^{19}$ Measurements were made three times each before and after wearing a Figure- 8 brace, and the average value of the resulting values was used as data.

\section{Statistical analysis}

The data collected in this study were compiled using SPSS 21.0 (SPSS Inc., Chicago, IL, USA). The general characteristics of the study subjects were calculated by calculating average and standard deviation using technical statistics. A regularity test was performed using the Shapiro-Wilk test method and all variables were normally distributed. FEV1, FVC, and balances resulting from the use of Figure- 8 brace in older women's groups of over 65 years and younger women in their 20s were compared with the preceding and subsequent changes through the paired t-tests , and the significance level was set at $\alpha=0.05$.

\section{Results}

\section{Comparison of results by wearing Figure- 8 brace in a group of elderly women}

The average of FEV1 was $2.10 \pm 0.20 \ell$ before wearing an Figure- 8 brace and $2.10 \pm 0.22 \ell$ after wearing it, with no statistically significant difference $(\mathrm{p}>0.05)$. The average of FVC was $2.27 \pm 0.22 \ell$ before wearing and the average of FVC was $2.29 \pm 0.21 \quad \ell$ after wearing, with no statistically significant difference $(\mathrm{p}>0.05)$. The distribution of the anterior foot pressure was $40.79 \pm 9.10 \%$ before wearing and $33.24 \pm 8.91 \%$ after wearing, with a statistically significant difference $(\mathrm{p}<0.05)$. The distribution of the posterior foot pressure was $57.97 \pm 4.22 \%$, and the posterior foot pressure was $61.41 \pm 5.05 \%$

Table 2. The change in FEV1, FVC and Balance according to Figure-8 brace in the Elderly

\begin{tabular}{|c|c|c|c|c|}
\hline & Pre & Post & $\mathrm{t}$ & $\mathrm{p}$ \\
\hline FEV1 (L) & $2.10 \pm 0.20$ & $2.10 \pm 0.22$ & -0.06 & 0.95 \\
\hline FVC (L) & $2.27 \pm 0.22$ & $2.29 \pm 0.21$ & -0.93 & 0.36 \\
\hline Anterior foot pressure (\%) & $40.79 \pm 9.10$ & $33.24 \pm 8.91$ & 3.62 & $0.00^{*}$ \\
\hline Posterior foot pressure (\%) & $59.25 \pm 9.04$ & $66.76 \pm 8.91$ & -3.67 & $0.00^{*}$ \\
\hline
\end{tabular}

Values are presented as mean \pm standard deviation, FEV1: forced expiratory volume in 1 second, FVC: forced vital capacity, * $p<0.05$. 
Table 3. The change in FEV1, FVC and Balance according to Figure-8 brace in the 20's

\begin{tabular}{lcccc}
\hline & Pre & Post & t & p \\
\hline FEV1 (L) & $2.78 \pm 0.12$ & $2.79 \pm 0.17$ & -0.39 & 0.71 \\
FVC (L) & $2.83 \pm 0.17$ & $3.14 \pm 0.20$ & -7.92 & $0.00 *$ \\
Anterior foot pressure (\%) & $39.69 \pm 5.57$ & $34.06 \pm 5.31$ & 11.88 & $0.00 *$ \\
Posterior foot pressure (\%) & $57.97 \pm 4.22$ & $61.41 \pm 5.05$ & -14.10 & $0.00 *$ \\
\hline
\end{tabular}

Values are presented as mean \pm standard deviation, FEV1: forced expiratory volume in 1 second, FVC: forced vital capacity, ${ }^{*} \mathrm{p}<0.05$.

after wearing Figure-8 brace, with a statistically significant difference $(\mathrm{p}<0.05)($ Table 2$)$.

\section{Comparison of results by wearing Figure- 8 brace within the 20-something female group}

Before wearing an eight-shaped shoulder aid, the average of FEV1 was $2.78 \pm 0.12 \mathrm{~L}$, and there was no statistically significant difference between $2.79 \pm 0.22 \mathrm{~L}$ after wearing it $(\mathrm{p}<0.05)$. The mean of FVC was $2.83 \pm 0.17 \mathrm{~L}$ prior to wearing and there was a statistically significant difference in FVC average of $3.14 \pm 0.20 \mathrm{~L}$ after wearing $(\mathrm{p}<0.05)$. The distribution of the anterior foot pressure was $39.79 \pm 5.57 \%$ before wearing and $34.06 \pm 5.31 \%$ after wearing, with a statistically significant difference $(\mathrm{p}<0.05)$. The distribution of the posterior foot pressure was $57.97 \pm 4.22 \%$, and the posterior foot pressure was $61.41 \pm 5.05 \%$ after wearing, with a statistically significant difference $(\mathrm{p}<0.05)$.

\section{Discussion}

Physiological vertebral curvature with age increase cause bending posture and are carried FHP, limiting spinal movement and movement, thereby physically limiting the expansion of the thorax and causing respiratory capacity and balance disorders. ${ }^{4,79,10,19}$

The purpose of this study was to investigate the effect of the Figure- 8 brace to mitigate FHP, which is caused by vertebral curvature, and the influence for the pulmonary function and postural balance by the brace. FEV1 and FVC were measured to determine changes in pulmonary function. The mean value of FEV1 and FVC in elderly women were not significantly different ( $p>0.05)$. There was no significant difference in female FEV1 among women in their 20s. However, the mean value of FVC differed significantly $(\mathrm{p}<0.05)$.

Lee and Chu showed that scapula descending exercise and stretching exercise of the pectoralis minor muscles with the shoulder brace extended the thoracic cage and improved the CVA. ${ }^{20}$ However, both groups did not show significant differences in FEV1 after the application of Figure-8 brace. In order to expand the thoracic cage and improve the CVA, the inspiration assistant muscles such as the scalene muscle, sternocleidomastoid (SCM) muscle, and upper trapezius muscle, should be improved. ${ }^{21,22}$ In this study, the application of Figure- 8 brace is thought to be the result of the failure to improve the inspiration assistant muscles. During inhalation, scalene and SCM muscles elevate sternum and ribs in the direction of the head, increase the volume of the thoracic cage. ${ }^{21,22}$ Kapreli et al. described that the anatomical changes in the cervical and thoracic vertebra causes the shortening and weakening of respiratory muscles. ${ }^{4,7}$ In addition, the failure of maximum exhalation during the measurement of FEV1 due to the inability to effective use the respiratory tract shall be considered. The smaller the angle of CVA, the more limited the expansion of the thoracic cage by thoracic kyphosis. ${ }^{20}$ In this study, the reason for the significant increase of FVC in women in their twenties compared to the elderly women is that, despite the decrease of endurance and flexibility by senescence, extension of thoracic cage in elderly women with small angle of CVA would be restricted due to thoracic kyphosis. In the FVC measurement, participant should inhale breath to the utmost limit, and exhale as fast and strong as possible for a long period of time. The value of FVC may depend on the movement of the diaphragm and the extent of thoracic cage expansion. ${ }^{12}$ Also, in order to achieve this forced expiration, the volume in the thoracic cavity must be reduced rapidly, and transversus abdominis, external oblique abdominal, internal oblique abdominal and rectus abdominis muscle must be actively contracted. ${ }^{23}$ For the women in their twenties, we can consider that contraction of transverse abdominis by the application of Figure- 8 brace caused elevation of diaphragm to the thoracic cavity by the increase of abdominal pressure, and facilitated exhalation of breath.

In the study of Bae et al. a male college student with FHP was measured by artificially taking a neutral head position, which resulted in significant results from FEV1 and FVC. ${ }^{24}$ However, only young women in their 20s showed significant results in this study. As such, the result is believed to be that the pressure of the strap to wear Figure- 8 brace limited the extension of the thoracic cage, failing to increase lung activity despite abnormal ver- 
tebral curvature improvement. In addition, in order to improvement of pulmonary function, the length-tension relationship of the respiratory muscles should be corrected, and the respiratory exercise program to rehabilitate respiratory muscles should be accompanied.

In FHP, the thoracic vertebra flexes forward and the center of gravity of the body moves forward. ${ }^{1,2}$ In this study, foot pressure was measured after wearing of Figure- 8 brace. The anterior foot pressure distribution was significantly decreased, and foot pressure distribution was significantly increased in both group of women, which is consistent with previous studies $(\mathrm{p}<0.05),{ }^{1,2,26,27}$ As the thoracic curvature increases, the center of gravity shifts, negatively affecting the balancing ability, and changes and imbalances in posture cause a decrease in activity ${ }^{10,11,25,26}$ A study by Sinaki and Lynn showed positive effects on balance and weight distribution by applying spine orthosis to elderly women with thoracic curvature, ${ }^{27}$ this suggested that spinal deformation and foot pressures were highly relevant.

As a result, in order to the application of Figure- 8 brace to effective improvement of FHP, the contact area between the orthosis and trunk shall be enlarged to effectively distribute the pressure applied to the trunk. The change of abdominal pressure by movement of diaphragm during breathing activates the truncal muscles, improves postural balance. However, irreversible pressure by the orthosis can impair the activation of trunk muscles and its effects. ${ }^{28}$

The fact that Figure- 8 brace itself cannot produce adequate level of respiratory muscle strength showed that physiological thoracic curvature and FHP due to gradual degeneration for a long time could not adapt to the body and could not produce adequate level of respiratory muscle strength. Therefore, in addition to sufficient intervention period for the application of Figure- 8 brace to affect pulmonary function, therapeutic interventions such as posture correction or breathing training to improve FHP will be needed. The limitations of this study are that it is difficult to generalize, because the study was conducted on women only, lack of comparison of mediation effect for a long time, and absence of controlled experiment with other posture or breathing training. In addition, the pulmonary function was measured in the sitting position without considering the increase in the FHP in the sitting position. Therefore, further study will be needed with the sufficient number of participants and duration of intervention, and combination of Figure- 8 brace and various physical therapeutic methods.

\section{References}

1. Katzman WB, Sellmeyer DE, Stewart AL et al. Changes in flexed posture, musculoskeletal impairments, and physical performance after group exercise in community-dwelling older women. Arch Phys Med Rehabil. 2007;88(2)192-9.

2. Briggs AM, van Dieën JH, Wrigley TV et al. Thoracic kyphosis affects spinal loads and trunk muscle force. Phys Ther. 2007;87(5):595-607.

3. Kim JS, Choi JH, Lee MY. Effect of self-postural control with visual feedback in the foot pressures in the subject with forward head posture. J Kor Phys Ther. 2017;29(4):153-7.

4. Kim EK, Lee DK. Effect of scapular brace on the pulmonary function and foot pressure of elderly women with forward head posture. J Kor Phys Ther. 2018:30(4):141-5.

5. Yip CH, Chiu TT, Poon AT. The relationship between head posture and severity and disability of patients with neck pain. Man Ther. 2008; 13(2):148-54.

6. Nishiwaki Y, Kikuchi Y, Araya K et al. Association of thoracic kyphosis with subjective poor health, functional activity and blood pressure in the community-dwelling elderly. Environ Health Prev Med. 2007;12(6): 246-50.

7. Kapreli E, Vourazanis E, Strimpakos N. Neck pain causes respiratory dysfunction. Med Hypothese. 2008:70(5):1009-13.

8. Priftis KN, Hager J, Vlachou M et al. Effects of bracing on lung function in idiopathic juvenile kyphosis. Pediatr Pulmonol. 2003;35(2):83-6.

9. Seacrist T, Saffioti J, Balasubramanian S et al. Passive cervical spine flexion: the effect of age and gender. Clin Biomech (Bristol, Avon). 2012; 27(4):326-33.

10. Aslan UB, Cavlak U, Yagci N et al. Balance performance, aging and falling: a comparative study based on a Turkish sample. Arch Gerontol Geriatr. 2008;46(3):283-92.

11. Ailon T, Shaffrey CI, Lenke LG et al. Progressive spinal kyphosis in the aging population. Neurosurgery. 2015;77(Suppl 4):S164-72.

12. Han JT, Go MJ, Kim YJ. Comparison of forced vital capacity and maximal voluntary ventilation between normal and forward head posture. J Korean Soc Phys Med. 2015:10(1):83-9.

13. Azadinia F, Kamyab M, Behtash H et al. The effects of two spinal orthoses on balance in elderly people with thoracic kyphosis. Prosthet Orthot Int. 2013;37(5):404-10.

14. Lee JH, Cynn HS, Yoon TL et al. The effect of scapular posterior tilt exercise, pectoralis minor stretching, and shoulder brace on scapular alignment and muscles activity in subjects with round-shoulder posture. J Electromyogr Kinesiol. 2015;25(1):107-14.

15. Salahzadeh Z, Maroufi N, Ahmadi A, et al. Assessment of forward head posture in female: Observational and photogrammetry methods. J Back Musculoskelet Rehabil. 2014;27(2):131-9.

16. Kim EK, Kim SG. The effect of an active vibration stimulus according to different shoulder joint angles on functional reach and stability of the shoulder joint. J Phys Ther Sci. 2016;28(3):747-51.

17. Cole AK, McGrath ML, Harrington SE et al. Scapular bracing and alteration of posture and muscle activity in overhead athletes with poor posture. J Athl Train. 2013;48(1):12-24

18. Lima IN, Fregonezi GA, Melo R et al. Acute effects of volume-oriented incentive spirometry on chest wall volumes in patients after a stroke. 
Respir Care. 2014;59(7):1101-7.

19. Pau M, Leban B, Collu G et al. Effect of light and vigorous physical activity on balance and gait of older adults. Arch Gerontol Geriatr. 2014; 59(3):568-73.

20. Lee MH, Chu M. Correlations between craniovertebral angle(CVA) and cardiorespiratory function in young-adults. J Korean Soc Phys Med. 2014:9(1):107-13.

21. Legrand A, Schneider E, Gevenois PA et al. Respiratory effects of the scalene and sternomastoid muscles in humans. J Appl Physiol. 2003; 94(4):1467-72.

22. Ovechkin A, Vitaz T, de Paleville DT et al. Evaluation of respiratory muscle activation in individuals with chronic spinal cord injury. Respir Physiol Neurobiol. 2010;173(2):171-8.

23. Hodges PW, Gandevia SC. Changes in intra-abdominal pressure during postural and respiratory activation of the human diaphragm. J Appl Physiol. 2000:89(3):967-76.
24. Bae WS, Lee KC, Park SW et al. Effect of the changes in forward head posture and neutral head posture on respiratory. KSIM. 2017;5(1):67-74.

25. Chang JS, Lee MH. The comparison of plantar foot pressure distribution in adult and elderly according obstacle heights. J Kor Phys Ther. 2014; 26(4):257-61.

26. Aslan UB, Cavlak U, Yagci N et al. Balance performance, aging and falling: a comparative study based on a Turkish sample. Arch Gerontol Geriatr. 2008;46(3):283-92.

27. Sinaki M, Lynn SG. Reducing the risk of falls through proprioceptive dynamic posture training in osteoporotic women with kyphotic posturing: a randomized pilot study. Am J Phys Med Rehabil. 2002;81(4):2416.

28. Kim YH, Jun SC, Jung DY. Jung et al. Biomechanical analysis of different thoracolumbar orthosis designs using finite element nethod. J. of RWEAT. 2012:6(1):45-50. 